\title{
Fabrication of PLGA coated silica nanorattle for controlling the drug release behavior
}

\author{
LI LinLin, ZHANG YanQi, HAO NanJing, CHEN Dong \& TANG FangQiong* \\ Laboratory of Controllable Preparation and Application of Nanomaterials, Technical Institute of Physics and Chemistry, Chinese Academy of \\ Sciences, Beijing 100190, China
}

Received March 6, 2012; accepted March 28, 2012; published online July 2, 2012

\begin{abstract}
Silica nanorattle with hollow and mesoporous structure has been proven to be an excellent drug carrier. However, how to control the drug release from silica nanorattle is still a challenge. In this study, we designed two methods, in-situ polymerization method and water in oil in water $(\mathrm{W} / \mathrm{O} / \mathrm{W})$ double emulsion method, to coat a nanosized poly(lactic-co-glycolic acid) (PLGA) layer onto the surface of silica nanorattle for controlling the drug release behavior. Hydrophobic antitumor drug docetaxel was loaded into the PLGA coated silica nanorattle (PLGA@SN). The drug release profile, cellular uptake and cytotoxicity on human liver cancer HepG2 cells were evaluated to prove that the PLGA layer plays an effective role in tuning the drug delivery.
\end{abstract}

silica nanorattle, PLGA, microemulsion, in-situ copolymerization, docetaxel, drug delivery

Citation: $\quad$ Li L L, Zhang Y Q, Hao N J, et al. Fabrication of PLGA coated silica nanorattle for controlling the drug release behavior. Chin Sci Bull, 2012, 57: 3631-3638, doi: 10.1007/s11434-012-5246-4

For the recent decade, mesoporous silica nanoparticles (MSNs) with high specific surface area, mesoporous structure and high biocompatibility have shown great potentials for their biomedical applications [1-5]. With these properties, MSNs have been proven to be versatile drug carriers for delivering a variety of therapeutic drugs with high drug loading capacity. Especially, mesoporous silica nanoparticles with hollow or rattle-type structure have vast empty spaces to accommodate large quantities of guest molecules, which provides opportunities for high drug loading [6,7].

After entering into body, the drug release profile from the nanocarriers would greatly influence the therapy efficacy. For controlling release profiles of the loaded therapeutic agents from the MSNs, stimuli-response drug delivery system (SRDDS) has been designed. Tanaka and co-workers designed a photo-controlled reversible drug release system based on MCM-41 type MSNs [8]. Lin and co-workers used surface-derivatized cadmium sulfide (CdS) nanocrystals as chemically removable caps to encapsulate the drug molecules in the mesopore of MSNs [9]. Other SRDDSs that can

*Corresponding author (email: tangfq@mail.ipc.ac.cn) response to $\mathrm{pH}$ [10], temperature [11], redox [9], magnetic field [12], and enzymes [13] have also been designed. With these effort, however, it is still great challenge to remotely manipulate the SRDDS in vivo by photo and magnetic field. It is also difficult for SRDDSs to sense the local microenvironment of disease foci, which may have very little difference with peripheral normal tissues in temperature, $\mathrm{pH}$, chemicals, and enzyme. Furthermore, for mesoporous silica nanoparticles with hollow structure, it presents an additional challenge: all of the pores connected to the hollow interior must be controlled. In hollow particles with multiple pore connectivity to the hollow center, absence of control would result in the loss of all of the contents of the particles. In our group, silica nanorattle with hollow and mesosporous structure synthesized by a simple and scalable structure-selective strategy has been proven to be promising candidate for drug delivery with high drug loading [13-18]. However, how to control the drug release from silica nanorattle is still a great challenge.

Organic nanocarriers such as liposomes, polymeric micelles and polymeric nanoparticles have a longer history of scientific research than MSNs [19,20]. Among various or- 
ganic nanobased drug delivery systems, Doxil [21] and Abraxane [22], approved by FDA, have produced exciting results in clinical applications. However, organic nanoparticles as drug carriers still have some serious limitations, which hinder their future industrial production and clinical translocation. First, the fabrication methods are generally complicated and difficult to be scaled-up. Second, the synthesized organic nanoparticles often have poor dispersion and monodispersity, which induce poor stability in body circulation. Third, the drug loading amount for organic nanoparticles are relatively low. Fourth, one organic nanocarrier could only load rather narrow kinds of therapeutic agents, which limits their application. All these issues come to be barriers for optimal therapy efficacy and future clinical translocation.

In this paper, we firstly coated a biocompatible copolymer, poly (lactic-co-glycolic acid) (PLGA) layers onto silica nanorattle $(\mathrm{SN})$ for controlling the drug release profile. PLGA is the most popular biodegradable polymer used in regenerative medicine, tissue engineering as well as drug delivery carriers [23-26]. The organic-inorganic nanocomposites could integrate the advantage of two different kinds of materials. The organic silica nanorattle with mesoporous and hollow structure has good dispersion and extraordinarily high drug loading amount. The PLGA layer on the surface of silica nanorattle can act as a gatekeeping layer to regulate the drug release from silica nanorattle. Two distinct methods, in-situ polymerization method and water in oil in water $(\mathrm{W} / \mathrm{O} / \mathrm{W})$ double emulsion method, were developed for coating PLGA onto the surface of silica nanorattle. The drug loading and release of docetaxel (Dtxl) from the PLGA coated silica nanorattle (PLGA@SN), the cellular uptake of the composite nanoparticle, and the cytotoxicity to HepG2 cancer cells were also investigated.

\section{Materials and methods}

\subsection{Materials}

Tetraethyl orthosilicate (TEOS), tetrahydrofuran (THF), ethanol, dichloromethane, poly(vinyl alcohol) (PVA), acetic ether, stannous octoate $\left(\mathrm{Sn}(\mathrm{Oct})_{2}\right)$ were obtained from Beijing Chemical Reagents Company (China). Fluorescein isothiocyanate (FITC) was from Sigma. D,L-lactide and glycolide were purchased from Beijing GLACO LTD. $N$-[3-(trimethoxysilyl) propyl] ethylenediamine (TSD) was from ACROS Co. PLGA copolymer $(75: 25)$ with molecular weight of $10 \mathrm{kDa}$ was purchased from Shandong Institute of Medical Instrument. Dtxl was from Sanwei Pharmaceutical., Shanghai.

\subsection{Synthesis of silica nanorattle}

SNs with particle size of $280 \mathrm{~nm}$ were synthesized as previous described $[6,13]$.

\subsection{Preparation of PLGA@SN by in-situ polymeriza- tion method}

Silica nanorattle was dispersed in $20 \mathrm{~mL}$ THF and sufficiently sonicated for $10 \mathrm{~min}$. The suspension was sealed and charged with $\mathrm{N}_{2}$ for $1 \mathrm{~h}$ under stirring. $\mathrm{Sn}(\mathrm{Oct})_{2}$ was injected into the reaction when the temperature was increased to $80^{\circ} \mathrm{C}$. Then, $D, L$-lactide and glycolide dissolved in THF was injected and the reaction was kept at $80^{\circ} \mathrm{C}$ for $24 \mathrm{~h}$ under $\mathrm{N}_{2}$. Finally, the formed composite nanoparticles, denoted as PLGA@SN-1, were harvested by repeated centrifugating and washing with ethanol.

\subsection{Preparation of PLGA@SN by $\mathrm{W}_{1} / \mathrm{O} / \mathrm{W}_{2}$ double emulsion method}

PLGA copolymer was dissolved in a mixed solution acetic ether and dichloromethane $(\mathrm{v} / \mathrm{v}=1: 1)$. SN or drug loaded $\mathrm{SN}$ was dispersed in $1 \mathrm{~mL}$ water. These two solutions were mixed, vortexed for $1 \mathrm{~min}$ and sonicated for $2 \mathrm{~min}$ at $100 \mathrm{~W}$ to form a microemulsion. Then, the microemulsion was added into 2\% PVA aqueous solution drop by drop and sonicated for $10 \mathrm{~min}$. After the organic solvents was removed in a rotary evaporator at $37^{\circ} \mathrm{C}$, the formed composite nanoparticles, denoted as PLGA@SN-2, were harvested by repeated centrifugating and washing with ethanol.

\subsection{Characterization of the nanoparticles}

Morphology and structure of the silica nanorattle and PLGA coated silica nanorattle were observed with JEOL-200CX transmission electron microscope (TEM). The zeta potentials, hydrodynamic diameter and poly index of nanoparticles were determined by dynamic light scattering (DLS) with Zetasizer 3000HSA (Malvern) in water at $25^{\circ} \mathrm{C}$. The nanoparticles were characterized using Varian Excalibur 3100 Fourier transform infrared spectrophotometer (FTIR) to detect the functional groups.

\subsection{Drug loading and release}

Docetaxel was loaded into silica nanorattle according to our previous report [13]. Briefly, to load Dtxl into silica nanorattle, $\mathrm{SN}$ was dispersed in a solution of Dtxl $(40 \mathrm{mg} / \mathrm{mL}$ in ethanol) and stirred for $24 \mathrm{~h}$, followed by centrifugating and washing extensively with PBS to obtain the drug-loaded SN. The same procedure was performed for SN@PLGA-1. Drug loaded SN, PLGA@SN-1 and PLGA@SN-2 were denoted as SN-Dtxl, PLGA@SN-Dtxl-1 and PLGA@SN-Dtxl-2, respectively. For drug release assay, the drug loaded SN and PLGA@SN samples were placed in dialysis tubing (10 kDa) and immersed in $20 \mathrm{~mL}$ of release medium, stirring at room temperature. The release medium $(8 \mathrm{~mL})$ was taken at given time intervals. The concentration of Dtxl was determined by UV/Vis spectroscopy measurements at a wavelength of $230 \mathrm{~nm}$. 


\subsection{Cell culture}

Human liver cancer HepG2 cells (ATCC) were maintained in high-glucose DMEM, supplemented with $10 \%$ FBS, 100 $\mathrm{U} / \mathrm{mL}$ penicillin, and $100 \mu \mathrm{g} / \mathrm{mL}$ streptomycin. All cultures were kept in an atmosphere of $5 \% \mathrm{CO}_{2}$ and $95 \%$ air at $37^{\circ} \mathrm{C}$.

\subsection{Fluorescent observation of cellular uptake}

For fluorescent observation, FITC doped SN (SN(FITC)) was synthesized as previously described [6,13]. PLGA coated SN(FITC)(PLGA@SN(FITC)-2) was prepared by $\mathrm{W} / \mathrm{O} / \mathrm{W}$ double emulsion following protocol in 1.4. The HepG2 cells were seeded on glass-bottom dishes $(35 \mathrm{~mm}$, kindly provided by MatTek Corporation). A final concentration of $100 \mu \mathrm{g} / \mathrm{mL}$ PLGA@SN(FITC)-2 was added to the cells and incubated for $1 \mathrm{~h}$. The cells were washed with PBS three times, fixed with $4 \%$ paraformaldehyde, and then stained with $10 \mu \mathrm{g} / \mathrm{mL}$ DAPI (2-(4-amidinophenyl)-6-indolecarbamidine dihydrochloride, Sigma). Micrographs were taken on a Nikon fluorescence microscope (Nikon Eclipse Ti-S, CCD: Ri1).

\subsection{Cell viability}

The cytotoxicity of free Dtxl, SN-Dtxl and PLGA@SN-2Dtxl on was evaluated by MTT viability assay. Dtxl was dissolved using DMSO, and the final concentration of DMSO in culture media was less than $0.5 \%$. The HepG2 cells were seeded at a density of 2000 cells/well on 96-well plates (Costar). After incubating the cells with free Dtxl, SN-Dtxl and PLGA@SN-2-Dtxl for 72 h, MTT (3-(4,5dimethylthiazol-2-yl)-2,5-diphenyltetrazolium bromide) (Sigma) (final concentration of $0.5 \mathrm{mg} / \mathrm{mL}$ ) was added to each well. After $4 \mathrm{~h}$ of incubation at $37^{\circ} \mathrm{C}$, colorimetric meas- urement was performed at $570 \mathrm{~nm}$ on a scanning multiwell spectrometer (Multiskan MK3 Thermo labsystems). Data were expressed as mean \pm standard deviation (SD) of at least six independent experiments.

\section{Result and discussion}

\subsection{Synthesis of PLGA@SN by in-situ polymerization method}

Ring-opening copolymerization is the most widely used method for the synthesis of biodegradable PLGA copolymer from $D, L$-lactide and glycolide with $\mathrm{Sn}(\mathrm{Oct})_{2}$ as catalyst. Using this method, herein we directly coated nanosized PLGA layer onto the surface of silica nanorattle. Figure 1(a) shows the scheme of preparation of PLGA@SN by in-situ polymerization method. Once synthesized, the silica nanorattle has positive surface with a $\zeta$-potential of from 20 to $40 \mathrm{mV}$ due to the existence of amino groups. $\mathrm{Sn}(\mathrm{Oct})_{2}$, which was approved food additive, was used as a catalyst. With appropriate amount of catalyst and monomer added into the suspension of silica nanorattle, the monomer would copolymerize and form an even PLGA layers on the surface of silica nanorattle. Figure 2(a) shows the TEM image of silica nanorattle with diameter of about $280 \mathrm{~nm}$. It can be observed that the SN has unique structural characteristics with hollow and mesoporous structure, narrow size distribution, and spherical morphology. As known, in the ringopening copolymerization reaction to form PLGA, concentration, purity and molar ratio of monomer, the concentration of catalyst, reaction temperature and time, etc. wound influence the molecular weight of resulting PLGA and the monomer conversation-rate [27]. Herein, we fixed the molar ratio of $D, L$-lactide to glycolide $(1: 1)$ and the mass ratio of monomer (total amount of $D, L$-lactide and glycolide) to $\mathrm{SN}$

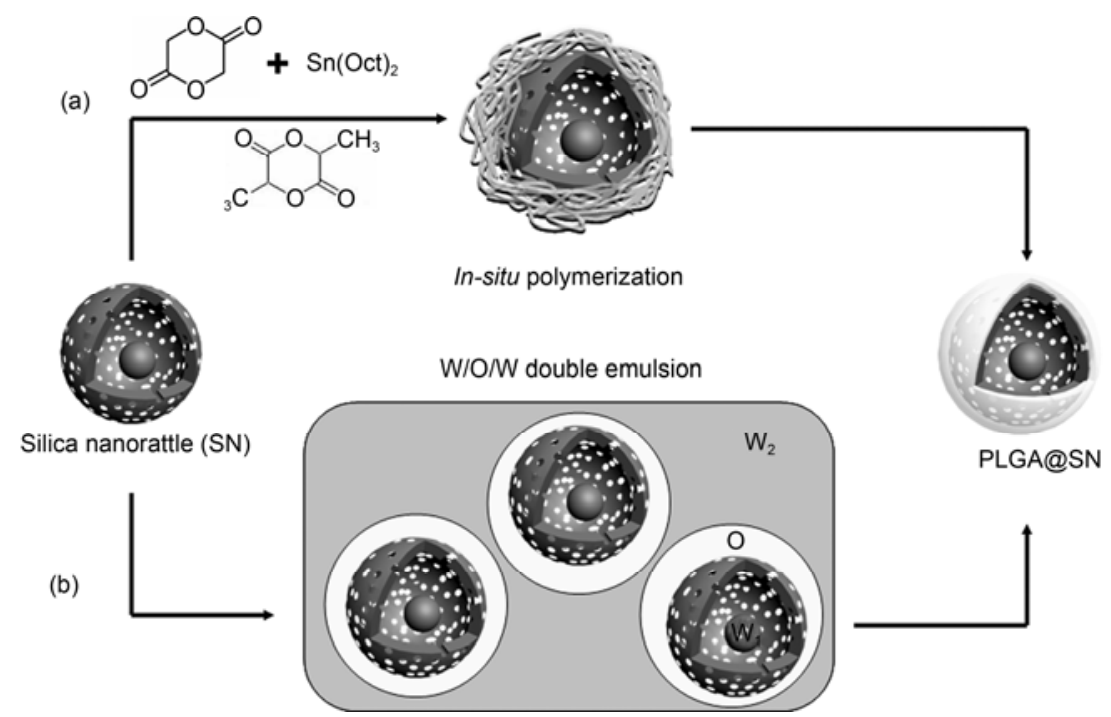

Figure 1 Schematic illustration of the preparation of PLGA@SN via in-situ polymerization method and W/O/W double emulsion method. 


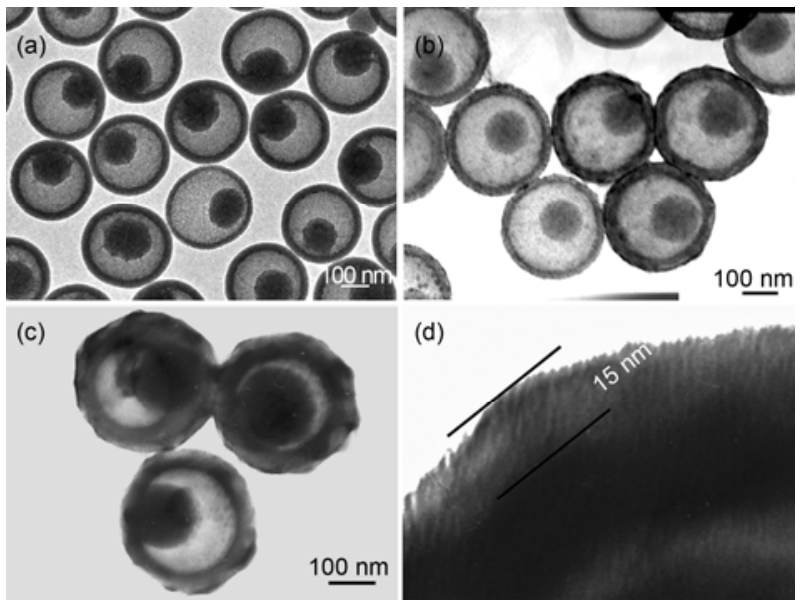

Figure 2 TEM images of silica nanorattle and the resulting produce via in-situ copolymerization method with different amount of catalyst (a); Sn $(\mathrm{Oct})_{2}:$ monomer: $\mathrm{SN}=2: 10: 10$ (b); $\mathrm{Sn}(\mathrm{Oct})_{2}:$ monomer:SN=10:10:10 (c), (d); $\mathrm{Sn}(\mathrm{Oct})_{2}:$ monomer: $\mathrm{SN}=100: 10: 10(\mathrm{~d})$.

(1:1), and researched the effect of concentration of catalyst on the synthesis of PLGA@SN.

When the mass ratio of $\mathrm{Sn}(\mathrm{Oct})_{2}:$ monomer: $\mathrm{SN}$ was 2 : $10: 10$, there formed a lot of island-like nanostructure on the surface of SNs (Figure 2(b)). When the amount of $\mathrm{Sn}(\mathrm{Oct})_{2}$ was increased and the mass ratio of $\mathrm{Sn}(\mathrm{Oct})_{2}:$ monomer:SN was $10: 10: 10$, there formed an integrated PLGA layer on the surface of silica nanorattle (Figure 2(c)). From the amplified TEM in Figure 2(d), a $15 \mathrm{~nm}$ PLGA layer on the surface of silica nanorattle could be observed. The resulting PLGA@SN composite nanoparticles have an average particle size of about $310 \mathrm{~nm}$. This PLGA@SN was denoted as PLGA@SN-1, which was used for drug delivery in following studies. When the amount of $\mathrm{Sn}(\mathrm{Oct})_{2}$ was further increased and the mass ratio of $\mathrm{Sn}(\mathrm{Oct})_{2}:$ monomer: $\mathrm{SN}$ reached $100: 10: 10$, copolymer could not be successfully coated on the surface of silica nanorattle (Figure S1). Most of the product from copolymerization reaction was 30-50 nm nanoparticles or some large irregular agglomeration. Thus, adjustment of the amount of the catalyst can control the morphology of formed nanocomposites. The mechanism is deduced as follows. When the amount of catalyst was low, the molecular weight of formed PLGA oligomer was accordingly low, which have good solubility in the reaction medium. Only some island-like nanostructure was deposited on the silica nanorattle (Figure 2(b)). When the catalyst is excessive, the reaction rate is high, which results in rapid self-seed nucleation of PLGA instead of coating onto the surface of silica nanorattle (Figure S1). Only when appropriate amount of catalyst was added, there could form a complete PLGA nanolayer on the surface of silica nanorattle. If needed, the mass ratio of monomer to silica nanorattle, and the molar ratio of $D, L$-lactide to glycolide could be tuned for tailoring the thickness of PLGA layer and the chemical constitution of PLGA for controlling the drug release.

\subsection{Synthesis of PLGA@SN by W/O/W double emul- sion method}

By the in-situ polymerization method, a complete PLGA layer could be coated onto the silica nanorattle. However, for some intrinsically instable therapeutic drugs, the reaction condition is relatively harsh, which demands that the drugs must be loaded into the nanoparticles after PLGA coating. As a substitute method, we also developed a mild $\mathrm{W}_{1} / \mathrm{O} / \mathrm{W}_{2}$ double emulsion solvent extraction method. As shown in Figure 1(b), the synthesis is consists of three steps. First, a $\mathrm{W}_{1} / \mathrm{O}$ emulsion is prepared by mixing the drug loaded silica nanorattle aqueous suspension with an organic phase containing the PLGA copolymer. Then, the primary emulsion is added into an aqueous phase $\left(\mathrm{W}_{2}\right)$ containing PVA as surfactants under sonication. Finally, after the organic solvent is evaporated in a rotary evaporator, the PLGA would solidify on the surface of silica nanorattle and form drug loaded PLGA@SN composite nanoparticles. The as-synthesized PLGA@SN had a diameter of about 304 nm, with a $12 \mathrm{~nm}$ PLGA layer (Figure 3 ). The resulting nanocomposite is denoted as PLGA@SN-2.

\subsection{Characterization of the nanoparticles}

To detect the PLGA coating, the zeta potentials of hydrodynamic diameter of nanoparticles before and after PLGA
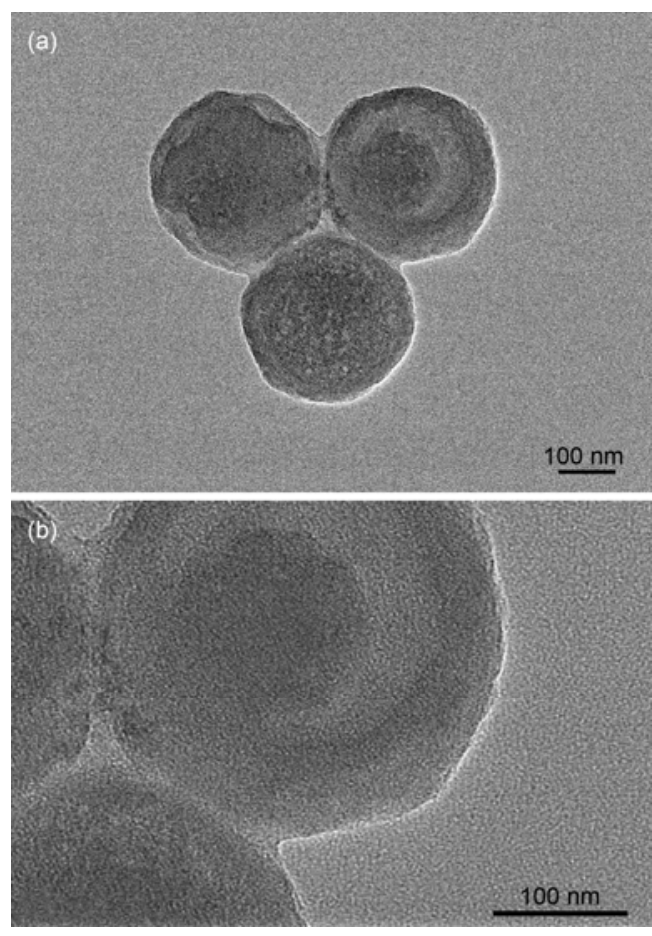

Figure 3 TEM images of PLGA@SN by $\mathrm{W}_{1} / \mathrm{O} / \mathrm{W}_{2}$ double emulsion solvent extraction method. 
coating were examined (Figure 4, Table 1). Due to the participation of amino-silane coupling agent TSD in the reaction to synthesize silica nanorattle, the as-synthesized silica nanorattle have a positive surface with zeta-potential of about $26 \mathrm{mV}$ (Figure 4(a)). In comparison, the PLGA@SN-1 synthesized from in-situ copolymerization method had a near-neutral surface with zeta-potential of about $5 \mathrm{mV}$, proving the successful coating of PLGA layer onto the silica nanorattle. PLGA@SN-2 via W/O/W double emulsion method had a negative surface potential with zeta-potential of about $-42 \mathrm{mV}$, because of the existence of PVA on the surface of resulting composite nanoparticles. The change of hydrodynamic diameter (HD) also proved the successful PLGA coating for both methods. The average HD determined by dynamic light scattering was increased from 294 $\mathrm{nm}$ for SN to $333.5 \mathrm{~nm}$ for PLGA@SN-1 and $340 \mathrm{~nm}$ for PLGA@SN-2, respectively. After PLGA coating, the poly index of the nanoparticles was slightly increased for both preparation methods, which still have good dispersion in aqueous solution without conglutination. The slight conglutination of nanoparticles observed in TEM image (Figure 3) is deduced to result from the harsh dry TEM environment with high vacuum, high temperature and high-intensity electron beam.

To further prove the presence of PLGA on the surface of particles, FTIR spectra were used to characterize the nanoparticles. As shown in Figure 5, compared with intact silica nanorattle, the peak at $1020-1250 \mathrm{~cm}^{-1}$ corresponding to $\mathrm{Si}-\mathrm{O}$ band and peak at $3300-3700 \mathrm{~cm}^{-1}$ corresponding to -OH bonds for PLGA@SN-1 and PLGA@SN-2 are weak- en. A strong peak at $1760 \mathrm{~cm}^{-1}$ appears for PLGA@SN synthesized from both methods due to stretching vibrations of $\mathrm{C}=\mathrm{O}$ bonds in PLGA. In addition, there are obvious peak at $1170 \mathrm{~cm}^{-1}$ for PLGA@SN-1 and PLGA@SN-2, which is deduced to be the amido bond group formed between the carboxyl group in PLGA and amino group at the surface of silica nanorattle. A peak at $1457 \mathrm{~cm}^{-1}$ is corresponding to the stretching vibrations of $\mathrm{C}-\mathrm{H}$ group in PLGA. In comparison, these peaks are not observed in intact silica nanorattle. These results indicate that PLGA layer were successfully coated onto the surface of the silica nanorattle for both methods.

\subsection{Drug release from intact SN and PLGA@SN}

In this research, we designed two methods to prepare PLGA @SN. The drug loading and release from the intact silica nanorattle and PLGA@SN was compared. A hydrophobic antitumor drug docetaxel, which has broad effectiveness against advanced breast carcinoma, ovarian cancer, prostate cancer, and nonsmall cell lung cancer, was used to compare the drug release property from intact silica nanorattle and PLGA@SN. Docetaxel was loaded into silica nanorattle according to our previous report. For PLGA@SN-2 synthesized from mild W/O/W double emulsion method, the docetaxel was loaded into silica nanorattle and then the PLGA was coated onto the silica nanorattle. However, considering the harsh reaction condition of in-situ polymerization and the intrinsically unstable property of the drug substance, docetaxel was loaded into PLGA@SN-1 after the nanocomposite
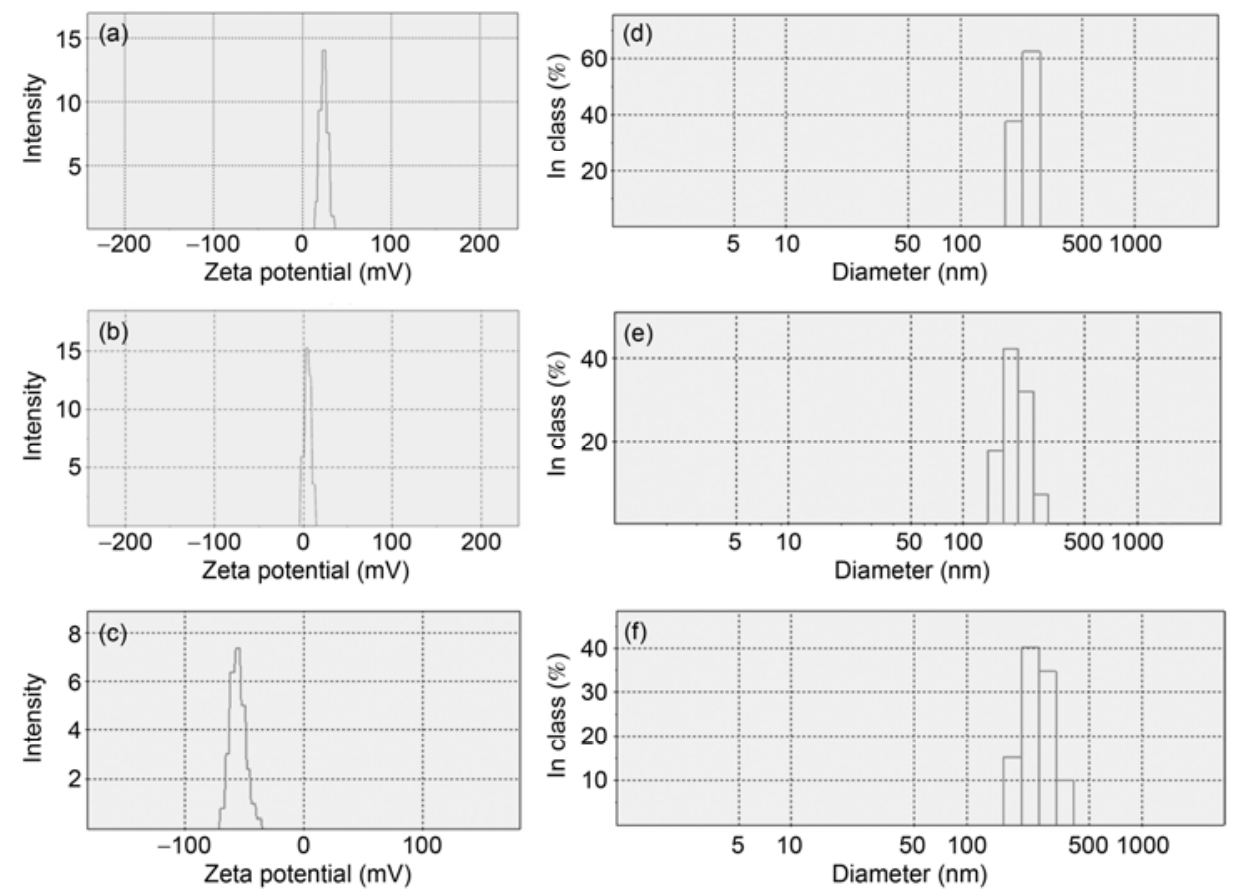

Figure 4 (a)-(c) Zeta potential and (d)-(f) hydrodynamic diameter of ((a), (d)) SN; (b), (e) PLGA@SN-1 from in-situ copolymerization method; (c), (f) PLGA@SN-2 by W/O/W double emulsion method. 
Table 1 Zeta potential, hydrodynamic diameter and poly index of SN, PLGA@SN-1 from in-situ copolymerization method and PLGA@SN-2 by $\mathrm{W} / \mathrm{O} / \mathrm{W}$ double emulsion method

\begin{tabular}{lrcc}
\hline & $\begin{array}{c}\text { Zeta potential } \\
(\mathrm{mV})\end{array}$ & $\begin{array}{c}\text { Hydrodynamic } \\
\text { diameter }(\mathrm{nm})\end{array}$ & Poly index \\
\hline SN & $26.4 \pm 2.4$ & 294.2 & 0.056 \\
PLGA@SN-1 & $5.3 \pm 1.4$ & 333.5 & 0.113 \\
PLGA@SN-2 & $-42.1 \pm 5.4$ & 340.2 & 0.155 \\
\hline
\end{tabular}

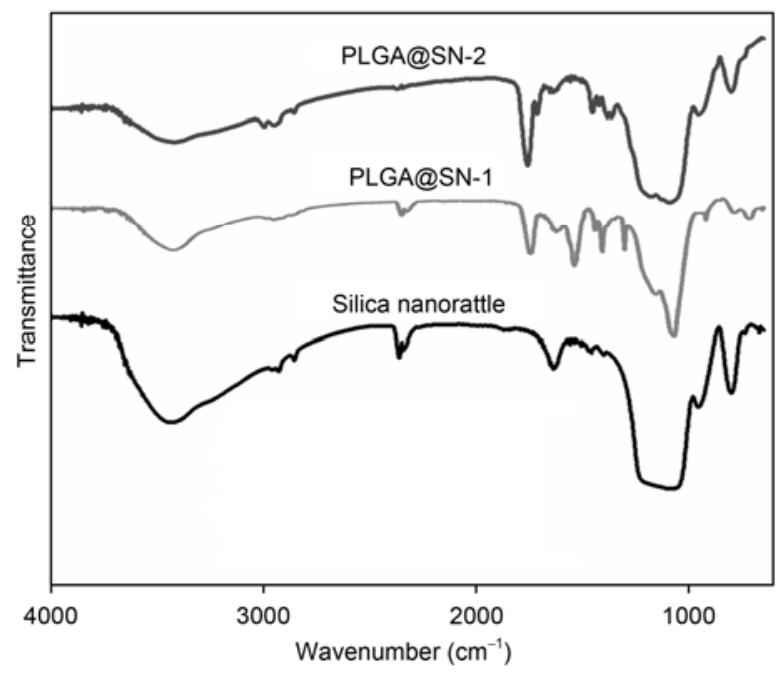

Figure 5 FTIR spectra of silica nanorattle and PLGA-coated silica nanorattle of PLGA@SN-1 synthesized by in-situ copolymerization method and PLGA@SN-1 synthesized by W/O/W double emulsion method.

was formed. Figure 6 shows the in vitro drug release profiles from intact silica nanorattle and the two kinds of PLGA@SN spheres in physiological pH and temperature. PLGA@SN-Dtxl-2 with pre-synthetic drug loading showed slower drug release rate compared with intact SN. The cumulative drug release from PLGA@ SN-Dtxl-2 at $1 \mathrm{~h}$ was about $9 \%$ of the total loaded drug, compared with $22 \%$ from intact SN-Dtxl. On the contrary, PLGA@SN-Dtxl-1 with post-synthetic drug loading had a faster release rate and a severe initial burst release (44\% release rate in the first hour). The cumulative drug release at $82 \mathrm{~h}$ was $75 \%, 92 \%$ and 56\% for SN-Dtxl, PLGA@SN-Dtxl-1, and PLGA@SNDtxl-2, respectively. This result demonstrates that coating a PLGA layer onto the surface of silica nanorattle (PLGA@ $\mathrm{SN}-2$ ) could effectively delay the drug release from silica nanorattle. However, if the drug is loaded into the integrate PLGA@SN nanocompisites (PLGA@SN-1) after synthesis, the drug could only penetrate in the outmost layer of PLGA, instead of entering into the inner hollow space of silica nanorattle, which determines the fast release rate than that released from intact silica nanorattle.

In comparison, these two distinct methods for coating PLGA layer onto silica nanorattle have respective characteristics. For in-situ polymerization method, it is convenient to control the thickness of PLGA layer and chemical constitution

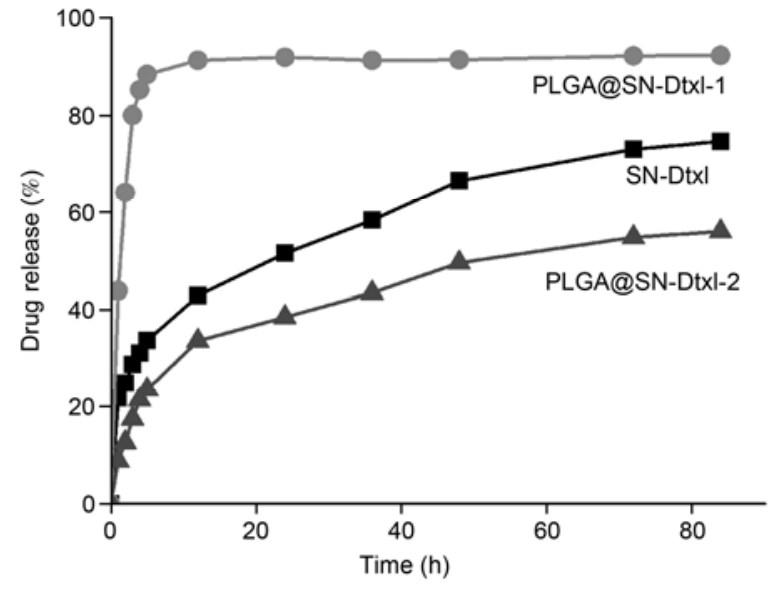

Figure 6 In vitro drug release profiles of docetaxel from intact silica nanorattle (SN-Dtxl), PLGA@SN-Dtxl-1 synthesized from in-situ copolymerization method and PLGA@SN-Dtxl-2 by W/O/W double emulsion method at physiological $\mathrm{pH}$ and temperature.

of PLGA. But it is not suitable for pre-loading of the temperature-sensitive drugs with high reaction temperature. For $\mathrm{W}_{1} / \mathrm{O} / \mathrm{W}_{2}$ double emulsion solvent extraction method with lower reaction temperature and shorter reaction time, the drugs could be pre-loaded into silica nanorattle. But it is relatively difficult to control the thickness of PLGA layer coating on the silica nanorattle.

\subsection{Cellular uptake}

Efficient cellular internalization of nanoparticles is necessary for intracellular drug delivery and efficient therapy. To monitor the trafficking of PLGA coated SN intracellularly, fluorescent labeled SN (SN(FITC)) were synthesized and coated with PLGA by W/O/W method. The resulting PLGA @ SN(FITC)-2 was incubated with HepG2 cells to observe the intracellular distribution. After incubating $100 \mu \mathrm{g} / \mathrm{mL}$ of PLGA@SN(FITC)-2 with HepG2 cells for $1 \mathrm{~h}$, the cells were fixed and nuclei-stained with DAPI (Figure 3(a)). PLGA@SN(FITC)-2 distributed in almost all of the cells throughout the entire cell cytoplasm (Figure 3(b)), demonstrating efficient cellular uptake. In some cells, there were isolated spots of green fluorescence showing higher fluorescence intensities, demonstrating the cluster of the composite nanoparticles.

\subsection{Cell viability}

With the sustained release property of PLGA@SN-Dtxl-2, we examined the cytotoxicity of free Dtxl, SN-Dtxl, and PLGA@SN-Dtxl-2 on HepG2 liver cancer cells. The cytotoxicity was evaluated via the MTT (3-(4,5-dimethylthiazol-2yl)-2,5-diphenyltetrazolium bromide) assay. HepG2 cells were incubated with a series of equivalent concentrations of free Dtxl, SN-Dtxl, or PLGA@SN-Dtxl-2 for 72 h, respectively. After 72 h incubation, free Dtxl, SN-Dtxl, and PLGA@SN- 

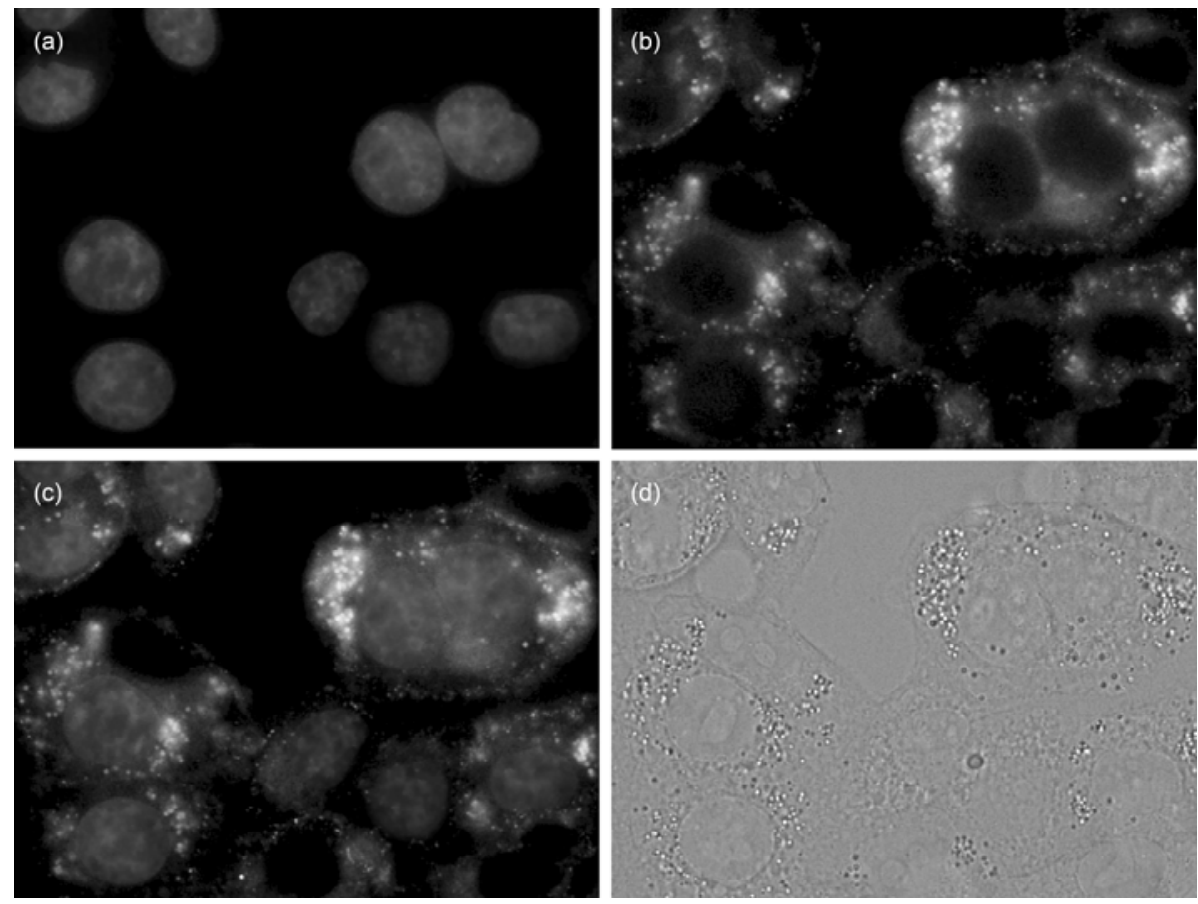

Figure 7 Uptake and subcellular localization of the PLGA@SN(FITC)-2 in HepG2 cells. The cells were incubated with $100 \mu \mathrm{g} / \mathrm{mL}$ of PLGA@SN(FITC)-2 for $1 \mathrm{~h}$, fixed, and then stained with DAPI. (a) Nuclear staining with DAPI; (b) fluorescence showing the location of PLGA@SN(FITC)-2; (c) overlaid image of (a) and (b); (d) corresponding transmission image.

Dtxl-2 in all the detected concentrations showed observable cytotoxicity (Figure 8). In comparison, SN-Dtxl and PLGA @ SN-Dtxl-2 showed obvious higher cytotoxicity than free Dtxl at equivalent drug concentration. Moreover, PLGA@ SN-Dtxl-2 exhibited obvious advantage over Dtxl in cytotoxicity in all concentrations especially at low concentration. The increased cytotoxicity for PLGA@SN-Dtxl-2 than SNDtxl may be attributed to the sustained release of the drug molecules from the nanoparticles and to increased cellular uptake of nanoparticles.

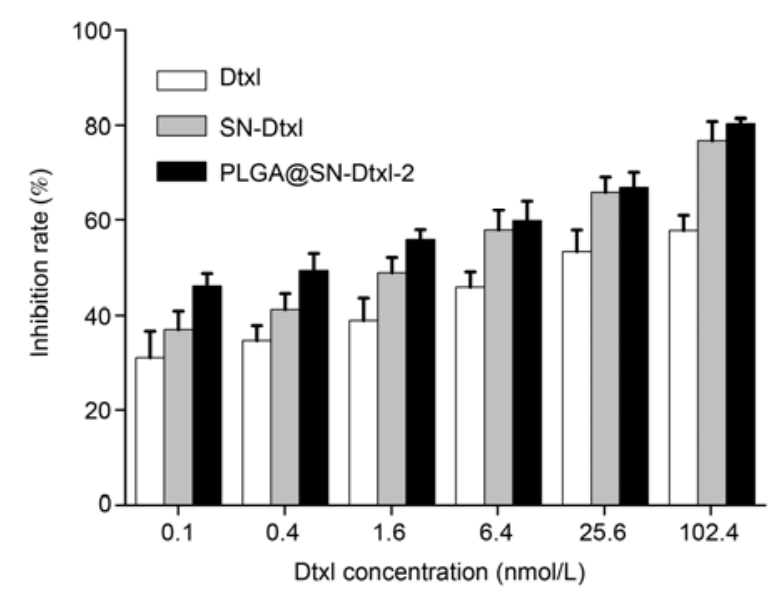

Figure 8 Cytotoxicity of Dtxl, SN-Dtxl, SN-PLGA-Dtxl-2 on Hep-G2 cells by MTT assays. Cell viability was detected with equivalent concentrations of Dtxl, SN-Dtxl, or PLGA@SN-Dtxl-2 for 72 h.

\section{Conclusion}

In conclusion, we have developed two distinct methods to prepare PLGA coated silica nanorattles for controlling the drug release behavior from the nanocarriers. The resulting PLGA@SN via W/O/W double emulsion method exhibits sustained drug release of docetaxel. The drug delivery system could be efficiently internalized by cells and showed advantage in killing cancer cells. It provides a promising way for controlling the drug release from mesoporous silica nanoparticles and other nanoparticles.

This work was supported by the National Natural Science Foundation of China (30900349, 81171454 and 81000667).

1 Tang F Q, Li L L, Chen D. Mesoporous silica nanoparticles: Synthesis, biocompatibility, and drug delivery. Adv Mater, 2012, doi: 10.1002/adma.201104763

2 Vallet-Regi M, Ramila A, del Real R P, et al. A new property of MCM-41: Drug delivery system. Chem Mater, 2001, 13: 308-311

3 Huang X L, Teng X, Chen D, et al. The effect of the shape of mesoporous silica nanoparticles on cellular uptake and cell function. Biomaterials, 2010, 31: 438-448

4 Huang X L, Li L L, Liu T L, et al. The shape effect of mesoporous silica nanoparticles on biodistribution, clearance, and biocompatibility in vivo. ACS Nano, 2011, 5: 5390-5399

5 Huang X L, Zhuang J, Teng X, et al. The promotion of human malignant melanoma growth by mesoporous silica nanoparticles through decreased reactive oxygen species. Biomaterials, 2010, 31: 6142-6153 
6 Chen D, Li L L, Tang F Q, et al. Facile and scalable synthesis of tailored silica "nanorattle" structures. Adv Mater, 2009, 21: 3804-3807

7 Tan L F, Chen D, Liu H Y, et al. A Silica nanorattle with a mesoporous shell: An ideal nanoreactor for the preparation of tunable gold cores. Adv Mater, 2010, 22: 4885-4889

8 Mal N K, Fujiwara M, Tanaka Y, et al. Photo-switched storage and release of guest molecules in the pore void of coumarin-modified MCM-41. Chem Mater, 2003, 15: 3385-3394

9 Lai C Y, Trewyn B G, Jeftinija D M, et al. A mesoporous silica nanosphere-based carrier system with chemically removable CdS nanoparticle caps for stimuli-responsive controlled release of neurotransmitters and drug molecules. J Am Chem Soc, 2003, 125: 44514459

10 Khashab N M, Trabolsi A, Lau Y A, et al. Redox- and pH-controlled mechanized nanoparticles. Eur J Org Chem, 2009, 2009: 1669-1673

11 Schlossbauer A, Dohmen C, Schaffert D, et al. pH-responsive release of acetal-linked melittin from SBA-15 mesoporous silica. Angew Chem-Int Ed, 2011, 50: 6828-6830

12 Hu S H, Liu T Y, Huang H Y, et al. Magnetic-sensitive silica nanospheres for controlled drug release, Langmuir. ACS J Surfaces Colloids, 2008, 24: 239-244

13 Li L L, Tang F Q, Liu H Y, et al. In vivo delivery of silica nanorattle encapsulated docetaxel for liver cancer therapy with low toxicity and high efficacy. ACS Nano, 2010, 4: 6874-6882

14 Li L L, Guan Y Q, Liu H Y, et al. Silica nanorattle-doxorubicinanchored mesenchymal stem cells for tumor-tropic therapy. ACS Nano, 2011, 5: 7462-7470

15 Liu T L, Li L L, Teng X, et al. Single and repeated dose toxicity of mesoporous hollow silica nanoparticles in intravenously exposed mice. Biomaterials, 2011, 32: 1657-1668

16 Liu H Y, Chen D, Li L L, et al. Multifunctional gold nanoshells on silica nanorattles: A platform for the combination of photothermal therapy and chemotherapy with low systemic toxicity. Angew ChemInt Ed, 2011, 50: 891-895

17 Liu H Y, Liu T L, Wu X L, et al. Targeting gold nanoshells on silica nanorattles: A drug cocktail to fight breast tumors via a single irradiation with near-infrared laser light. Adv Mater, 2012, doi: 10.1002/ adma.201103343

18 Liu T L, Li L L, Fu C H, et al. Pathological mechanisms of liver injury caused by continuous intraperitoneal injection of silica nanoparticles. Biomaterials, 2012, 33: 2399-2407

19 Davis M E, Chen Z, Shin D M. Nanoparticle therapeutics: An emerging treatment modality for cancer. Nat Rev Drug Discovery, 2008, 7: 771-782

20 Wagner V, Dullaart A, Bock A K, et al. The emerging nanomedicine landscape. Nat Biotechnol, 2006, 24: 1211-1217

21 Cianfrocca M E, Kaklamani V, Rosen S, et al. A phase I trial of a pegylated liposomal anthracycline (doxil (TM)) and lapatinib combination in the treatment of metastatic breast cancer: Doseescalation results of an anthracycline and lapatinib combination trial. Cancer Res, 2009, 69: 678s

22 Tishler R B, Lorch J, Sher D, et al. A phase I/II study of concurrent abraxane, carboplatin and IMRT for locally advanced squamous cell cancer of the head and neck (LASCCHN). Int J Radiat Oncol Biol Phys, 2011, 81: S494

23 Wang Z, Zhang Z, Zhang J C, et al. Distribution of bone marrow stem cells in large porous polyester scaffolds. Chin Sci Bull, 2009, 54: 2968-2975

24 Chang G T, Ci T Y, Yu L, et al. Enhancement of the fraction of the active form of an antitumor drug topotecan via an injectable hydrogel. J Control Release, 2011, 156: 21-27

$25 \mathrm{Wu} \mathrm{L}$ B, Ding J D. In vitro degradation of three-dimensional porous poly(D,L-lactide-co-glycolide) scaffolds for tissue engineering. Biomaterials, 2004, 25: 5821-5830

26 Chang G T, Li C, Lu W Y, et al. N-Boc-Histidine-Capped PLGAPEG-PLGA as a smart polymer for drug delivery sensitive to tumor extracellular pH. Macromol Biosci, 2010, 10: 1248-1256

27 Gou M, Wei X, Men K, et al. PCL/PEG copolymeric nanoparticles: Potential nanoplatforms for anticancer agent delivery. Curr Drug Targets, 2011, 12: 1131-1150

Open Access This article is distributed under the terms of the Creative Commons Attribution License which permits any use, distribution, and reproduction in any medium, provided the original author(s) and source are credited.

\section{Supporting Information}

Figure S1 TEM images of resulting produce via in-situ copolymerization method with $\operatorname{Sn}(\mathrm{Oct})_{2}: \operatorname{monomer}: \mathrm{SN}=100: 10: 10$.

The supporting information is available online at csb.scichina.com and www.springerlink.com. The supporting materials are published as submitted, without typesetting or editing. The responsibility for scientific accuracy and content remains entirely with the authors. 